\title{
Fractional supersymmetry and hierarchy of shape invariant potentials
}

\author{
M. Daoud ${ }^{12}$ and M. R. Kibler ${ }^{3}$ \\ Université de Lyon, \\ IPNL, université Lyon 1, CNRS/IN2P3, \\ Villeurbanne, F-69622, France
}

Fractional supersymmetric quantum mechanics is developed from a generalized WeylHeisenberg algebra. The Hamiltonian and the supercharges of fractional supersymmetric dynamical systems are built in terms of the generators of this algebra. The Hamiltonian gives rise to a hierarchy of isospectral Hamiltonians. Special cases of the algebra lead to dynamical systems for which the isospectral supersymmetric partner Hamiltonians are connected by a (translational or cyclic) shape invariance condition.

\footnotetext{
${ }^{1}$ Permanent address: Département de Physique, Faculté des Sciences, Université Ibn Zohr, Agadir, Morocco

${ }^{2}$ Electronic mail: m_daoud@hotmail.com

${ }^{3}$ Electronic mail: m.kibler@ipnl.in2p3.fr
} 


\section{INTRODUCTION}

Supersymmetry was initially introduced in high energy physics, as a kind of symmetry between bosons and fermions, to describe fundamental interactions of Nature in an unified way (e.g., see Ref. 1). Supersymmetry cannot be an exact symmetry. In order to understand supersymmetry breaking in quantum field theory, Witten studied supersymmetric quantum mechanics (SUSYQM). ${ }^{2}$ In the present days, SUSYQM turns out to be a powerful tool to investigate integrability in quantum mechanics. ${ }^{3-5}$ In this connection, the concept of shape invariant potential was introduced by Gendenshtein. ${ }^{6}$ This concept is especially useful to determine the spectrum of exactly solvable potentials. Indeed, for a given solvable potential, shape invariance implies integrability. It is now well-known there are three kinds of shape invariant potentials, namely, translational shape invariant potentials, ${ }^{7,8}$ scaling shape invariant potentials ${ }^{9,10}$ and cyclic shape invariant potentials. ${ }^{11,12}$

For any exactly solvable Hamiltonian (shape invariant or not), SUSYQM provides us with a process to generate a supersymmetric partner Hamiltonian. This process can be used successively to span a hierarchy of isospectral Hamiltonians. ${ }^{5}$

The aim of this work is to study shape invariant potentials together with the generation of a hierarchy of isospectral superpartner Hamiltonians in the framework of fractional SUSYQM of order $k(k=3,4, \cdots)$.

In general, to pass from ordinary SUSYQM to fractional SUSYQM of order $k$ (abbreviated as $k$-SUSYQM in the following), it is necessary to replace the $Z_{2}$-grading of the relevant Hilbert space by a $Z_{k}$-grading. This amounts either to replace a fermionic degree of freedom by a para-fermionic ${ }^{13-18}$ one, of order $k-1$, or to introduce $k$-fermions, ${ }^{19-22}$ which are objects interpolating between bosons and fermions. Quantum groups, with the deformation parameter being a root of unity, play also an important role in the development of $k$-SUSYQM. ${ }^{23,31}$ On the other hand, a realization of bosonized $k$-SUSYQM can be developed owing to the introduction of a Klein operator of order $k\left(K^{k}=1\right)$ which induces a $Z_{k}$-grading. ${ }^{18,32}$ In this direction, a relation exists between $k$-SUSYQM and hidden supersymmetric structures. ${ }^{33-36}$

The approach of $k$-SUSYQM developed in the present paper took its origin in Ref. 22 (see also Refs. 37-39 for some similar developments). It is based on a $Z_{k}$-graded WeylHeisenberg algebra $W_{k}$. Section 3 deals with algebra $W_{k}$ and its use for generating a family of $k$ isospectral Hamiltonians. In Sections 4 and 5, some specific Hamiltonians (with translational shape invariant potentials or cyclic shape invariant potentials) corresponding to particular cases of the algebra $W_{k}$ are studied. We will start in Section 2 with some preliminaries and motivations.

Throughout the present paper, $[A, B]$ and $\{A, B\}$ stand for the commutator and the anti-commutator of the operators $A$ and $B$, respectively. The operator $A^{\dagger}$ denotes the adjoint of $A$. The symbol $\delta$ is the Kronecker delta. Many quantities are defined modulo $k\left(\Pi_{k} \equiv \Pi_{0}, H_{k} \equiv H_{0}, F_{k} \equiv F_{0}\right.$ and $\left.V_{k} \equiv V_{0}\right)$. As usual, $f \circ g(x)=f(g(x))$ for two functions $f$ and $g$. We shall use the convention according to which $\sum_{i=a}^{b} x(i)=0$ when $b<a$ and the symbols $S_{0}$ and $S_{1}$ for denoting the sets $\{0,1, \cdots, k-1\}$ and $\{1,2, \cdots, k\}$, respectively. 


\section{PRELIMINARIES AND MOTIVATIONS}

For the purpose to establish our notations and to present our motivations, we shall begin with a brief review of ordinary SUSYQM, corresponding to $k=2$, and of shape invariance (for more details, see Refs. 3-5).

\subsection{Ordinary Supersymmetric Quantum Mechanics}

Let us start with ordinary SUSYQM. A supersymmetric dynamical system is defined by a triplet $\left(H, Q_{+}, Q_{-}\right)_{2}$ of linear operators acting on a $Z_{2}$-graded Hilbert space $\mathcal{H}$ and satisfying the following relations

$$
\begin{gathered}
H=H^{\dagger}, \quad Q_{-}=Q_{+}^{\dagger}, \quad Q_{ \pm}^{2}=0 \\
\left\{Q_{-}, Q_{+}\right\}=H, \quad\left[H, Q_{ \pm}\right]=0 .
\end{gathered}
$$

The operators $Q_{+}$and $Q_{-}$are the supercharges of the system. The self-adjoint operator $H$, the supersymmetric Hamiltonian for the (one-dimensional) system, can be written as

$$
H=H_{0}+H_{1},
$$

where $H_{0}$ and $H_{1}$ act on the states $|n, 0\rangle$ and $|n, 1\rangle$ of grading 0 and 1 , respectively. These states span the Hilbert space

$$
\mathcal{H}=\{|n, s\rangle: n \text { ranging; } s=0,1\} .
$$

We shall assume that there is no supersymmetry breaking. In this situation, the Hamiltonians $H_{0}$ and $H_{1}$ are isospectral except that the ground state of $H_{0}$ has no supersymmetric partner in the spectrum of $H_{1}$.

Now suppose that $H_{0}$ has $p$ states $|n, 0\rangle$ with $n=0,1, \cdots, p-1(p \geq 2)$. From the Hamiltonian $H_{1}$ with $p-1$ states $|n, 1\rangle(n=1,2, \cdots, p-1)$, we can find a supersymmetric partner $H_{2}$ with $p-2$ states $|n, 2\rangle(n=2,3, \cdots, p-1)$ and we can repeat this process to generate a hierarchy of $p$ Hamiltonians $H_{0}, H_{1}, \cdots, H_{p-1}$. The Hamiltonian $H_{m}$ $(0<m<p)$ has the same energy spectrum than $H_{0}$ except that the $m-1$ first energies of $H_{0}$ do not occur in the spectrum of $H_{m}$. This result remains valid when $p$ goes to infinity.

We can then ask the following question. What happens when we go from ordinary SUSYQM to $k$-fractional SUSYQM (with $k=3,4, \cdots$ )? We shall answer this question by showing that a hierarchy of isospectral Hamiltonians $H_{0}, H_{1}, \cdots, H_{k-1}$ can be constructed from a single Hamiltonian $H_{0}$ by making use of a $Z_{k}$-graded Weyl-Heisenberg algebra. This construction shall be achieved without a repetition process of the type $H_{0} \rightarrow H_{1}$, $H_{1} \rightarrow H_{2}, \cdots, H_{k-2} \rightarrow H_{k-1}$ as used in ordinary SUSYQM.

\subsection{Shape Invariance}

It is known that there exists a set of exactly solvable potentials characterized by an integrability condition known as shape invariance condition. ${ }^{3,5,6}$ In connection with ordinary SUSYQM, this shape invariance condition leads in an easy way to the spectrum of any invariant shape potential. 
More precisely, let us consider the partner potentials $V_{0}\left(x, a_{0}\right)$ and $V_{1}\left(x, a_{0}\right)$ associated with the supersymmetric Hamiltonians $H_{0}$ and $H_{1}$ such that

$$
H_{s}=-\frac{d^{2}}{d x^{2}}+V_{s}\left(x, a_{0}\right), \quad s=0,1
$$

where $a_{0}$ is a set of real parameters. The shape invariance condition is defined by

$$
V_{1}\left(x, a_{0}\right)=V_{0}\left(x, a_{1}\right)+R\left(a_{0}\right),
$$

where $a_{1}=h\left(a_{0}\right)$ corresponds to a reparametrisation in $V_{0}$ and $R\left(a_{0}\right)$ is a constant. The shape invariance condition immediately yields the energies and wavefunctions of $H_{0} \cdot{ }^{3,5}$ One obtain the energies $E_{n, 0}$ of $H_{0}$

$$
E_{n, 0}=\sum_{l=0}^{n-1} R\left(a_{l}\right), \quad n \geq 1,
$$

where

$$
a_{l}=h^{(l)}\left(a_{0}\right)=h \circ h \circ \cdots \circ h\left(a_{0}\right), \quad l \text { times. }
$$

(We take $E_{0,0}=0$.) The three kinds of shape invariance mentioned in the introduction correspond to $a_{1}=a_{0}+\alpha$ with $\alpha \in \mathbf{R}, a_{1}=\beta a_{0}$ with $0<\beta<1$, and $a_{1}=h\left(a_{0}\right)$ with $h^{(l)}\left(a_{0}\right)=a_{0}$ for translational, ${ }^{7,8}$ scaling, ${ }^{9,10}$ and cyclic ${ }^{11,12}$ shape invariance, respectively.

Another motivation for this work is to show that the isospectral Hamiltonians obtained from $k$-SUSYQM are connected through shape invariance. In this respect, we shall use some specific realizations of the $Z_{k}$-graded Weyl-Heisenberg algebra $W_{k}$ in order to generate a hierarchy of Hamiltonians subjected to translational or cyclic shape invariance.

\section{FRACTIONAL SUPERSYMMETRIC QUANTUM ME- CHANICS}

\subsection{Definition}

Let us go now to $k$-SUSYQM. A $k$-fractional supersymmetric dynamical system is defined by a triplet $\left(H, Q_{+}, Q_{-}\right)_{k}$ of operators satisfying the following relations ${ }^{13-16,22}$

$$
\begin{gathered}
H=H^{\dagger}, \quad Q_{-}=Q_{+}^{\dagger}, \quad Q_{ \pm}^{k}=0 \\
\sum_{s=0}^{k-1} Q_{-}^{k-1-s} Q_{+} Q_{-}^{s}=Q_{-}^{k-2} H, \quad\left[H, Q_{ \pm}\right]=0
\end{gathered}
$$

where $k=3,4, \cdots$. The Hamiltonian $H$ and the supercharges $Q_{ \pm}$of the dynamical system are linear operators acting on a Hilbert space $\mathcal{H}$,

$$
\mathcal{H}=\bigoplus_{s=0}^{k-1} \mathcal{H}_{s}
$$

which is $Z_{k}$-graded in view of the relations $Q_{ \pm}^{k}=0$. It is to be observed that Eq. (2) works equally well in the case $k=2$ corresponding to ordinary SUSYQM. 


\subsection{Generalized Weyl-Heisenberg algebra}

Following Ref. 22, we consider the generalized Weyl-Heisenberg algebra $W_{k}$, with $k \in$ $\mathbf{N} \backslash\{0,1\}$, spanned by the four linear operators $X_{+}, X_{-}, N$ and $K$ acting on the space $\mathcal{H}$ and satisfying

$$
\begin{gathered}
X_{-}=X_{+}^{\dagger}, \quad N=N^{\dagger}, \quad K K^{\dagger}=K^{\dagger} K=1, \quad K^{k}=1, \\
{\left[X_{-}, X_{+}\right]=\sum_{s=0}^{k-1} f_{s}(N) \Pi_{s}, \quad\left[N, X_{-}\right]=-X_{-}, \quad\left[N, X_{+}\right]=X_{+},} \\
K X_{+}-q X_{+} K=0, \quad K X_{-}-p X_{-} K=0, \quad[K, N]=0,
\end{gathered}
$$

where $q$ and $p$ are roots of unity with

$$
q=\mathrm{e}^{\frac{2 \pi \mathrm{i}}{k}}, \quad p=\mathrm{e}^{-\frac{2 \pi \mathrm{i}}{k}} .
$$

In Eq. (3), the functions $f_{s}: N \mapsto f_{s}(N)$ of the number operator $N$ are such that

$$
f_{s}(N)=f_{s}(N)^{\dagger} \text {. }
$$

Furthermore, the operators $\Pi_{s}$ are defined in terms of the Klein or grading operator $K$ as

$$
\Pi_{s}=\frac{1}{k} \sum_{t=0}^{k-1} p^{s t} K^{t}, \quad s \in S_{0} .
$$

It is easy to check that

$$
\Pi_{s}=\Pi_{s}^{\dagger}, \quad \sum_{s=0}^{k-1} \Pi_{s}=1, \quad \Pi_{s} \Pi_{t}=\delta_{s, t} \Pi_{s} .
$$

Consequently, the operators $\Pi_{s}$ are projection operators for the cyclic group $Z_{k}$. It can be proved that they satisfy

$$
\Pi_{s} X_{+}=X_{+} \Pi_{s-1} \Longleftrightarrow X_{-} \Pi_{s}=\Pi_{s-1} X_{-} .
$$

Note that the operators $X_{+}$and $X_{-}$can be considered as generalized creation and annihilation operators, respectively.

It should be realized that, for fixed $k$, Eq. (3) defines indeed a family of generalized Weyl-Heisenberg algebras $W_{k}$. The various members of the family are distinguihed by the various sets $\left\{f_{s}\right\} \equiv\left\{f_{s}(N): s \in S_{0}\right\}$.

\subsection{Realization of $k$-SUSYQM}

We can use the generators of $W_{k}$ for obtaining a realization of $\left(H, Q_{+}, Q_{-}\right)_{k}$.

First, we take the supercharge operators $Q_{ \pm}$(see Eq. (2)) in the form

$$
Q_{-}=X_{-}\left(1-\Pi_{1}\right), \quad Q_{+}=X_{+}\left(1-\Pi_{0}\right) .
$$

It can be proved that they satisfy the Hermitean conjugation property $Q_{-}=Q_{+}^{\dagger}$ and the $k$-nilpotency property $Q_{ \pm}^{k}=0$. Note that there are $k$ equivalent definitions of type (4) corresponding to the $k$ circular permutations of $0,1, \cdots, k-1$. 
Second, the $k$-fractional supersymmetric Hamiltonian $H$, satisfying (2) and compatible with (4), takes the form ${ }^{22}$

$$
H=(k-1) X_{+} X_{-}-\sum_{s=3}^{k} \sum_{t=2}^{s-1}(t-1) f_{t}(N-s+t) \Pi_{s}-\sum_{s=1}^{k-1} \sum_{t=s}^{k-1}(t-k) f_{t}(N-s+t) \Pi_{s},
$$

in terms of $X_{+} X_{-}, \Pi_{s}$ and $f_{s}$. In addition, it can be shown that the operator $H$ can be decomposed as

$$
H=\sum_{s=1}^{k} H_{s} \Pi_{s}=\sum_{s=0}^{k-1} H_{k-s} \Pi_{k-s}
$$

where

$$
H_{s}=(k-1) X_{+} X_{-}-\sum_{t=2}^{k-1}(t-1) f_{t}(N-s+t)+(k-1) \sum_{t=s}^{k-1} f_{t}(N-s+t), s \in S_{1} .
$$

As an important result, it can be proved, from $\left[H, Q_{ \pm}\right]=0$, that the $k$ operators $H_{k} \equiv H_{0}$, $H_{k-1}, \cdots, H_{1}$ constitute a hierarchy of isospectral Hamiltonians. Therefore, the spectra of $H_{1}, H_{2}, \cdots, H_{k-1}$ can be deduced from the spectrum of $H_{0}$.

\subsection{Representation of $W_{k}$}

Let us now examine the action of $X_{+}, X_{-}, N$ and $K$ on each subspace

$$
\mathcal{H}_{s}=\{|n, s\rangle: n \text { ranging }\}
$$

of $\mathcal{H}$ ( $n$ can take a finite or infinite number of values according to whether as $\mathcal{H}_{s}$ is of finite or infinite dimension). For this purpose, we introduce the structure functions $F_{s}: N \mapsto F_{s}(N)$ through

$$
X_{+} X_{-}=\sum_{s=0}^{k-1} F_{s}(N) \Pi_{s}, \quad X_{-} X_{+}=\sum_{s=0}^{k-1} F_{s+1}(N+1) \Pi_{s} .
$$

In view of Eq. (3), we have the recurrence relation

$$
F_{s+1}(n+1)-F_{s}(n)=f_{s}(n), \quad F_{s}(0)=0 .
$$

Then, we can take 22

$$
\begin{gathered}
X_{+}|n, s\rangle=\sqrt{F_{s+1}(n+1)}|n+1, s+1\rangle, \quad s \neq k-1, \\
X_{+}|n, s\rangle=\sqrt{F_{s+1}(n+1)}|n+1,0\rangle, \quad s=k-1, \\
X_{-}|n, s\rangle=\sqrt{F_{s}(n)}|n-1, s-1\rangle, \quad s \neq 0, \\
X_{-}|n, s\rangle=\sqrt{F_{s}(n)}|n-1, k-1\rangle, \quad s=0, \\
N|n, s\rangle=n|n, s\rangle, \quad K|n, s\rangle=q^{s}|n, s\rangle
\end{gathered}
$$

for the action of $X_{+}, X_{-}, N$ and $K$ on space $\mathcal{H}_{s}$. Relations (8) define a representation of $W_{k}$.

In the following, we shall consider two special cases of $W_{k}$ : (i) The case where $f_{s}(N)$ is independent of $s$ (see Section 4) and (ii) The case where $f_{s}(N)$ is independent of $N$ (see Section 5). 


\section{TRANSLATIONAL SHAPE INVARIANT POTENTIALS}

\subsection{Structure function}

In this section, we assume that $f_{s}(N)$ is independent of $s$ and linear in $N$. More precisely, we take

$$
f_{s}(N)=a N+b \Rightarrow\left[X_{-}, X_{+}\right]=a N+b,
$$

with strictly positive eigenvalues, where $a$ and $b$ are two real parameters. Thus, from Eq. (7) we have

$$
X_{+} X_{-} \equiv F(N, a, b)
$$

where

$$
F(N, a, b)=\frac{1}{2} a N(N-1)+b N .
$$

The non-linear spectrum of $X_{+} X_{-}$is then given by

$$
X_{+} X_{-}|n, s\rangle=\left[\frac{1}{2} a n(n-1)+b n\right]|n, s\rangle .
$$

For either $a=0$ and $b>0$ or $a>0$ and $b \geq 0$, the spectrum of $X_{+} X_{-}$is infinitedimensional and does not present degeneracies. For $a<0$ and $b \geq 0$, the spectrum of $X_{+} X_{-}$is finite-dimensional with $n=0,1, \cdots, E\left(-\frac{b}{a}\right)$ and all the states are non-degenerate.

It is possible to find a realization of each of the three cases just described in terms of an exactly solvable dynamical system in a one-dimensional space, with coordinate $x$, and characterized by a potential $V(x, a, b)$. As a matter of fact, we have:

(i) $a=0$ and $b=1$ correspond to the harmonic oscillator potential

$$
V_{h o}(x, 0,1)=x^{2},
$$

with an infinite non-degenerate spectrum $(n \in \mathbf{N})$.

(ii) $a=2$ and $b=u+v+1$, with $u>1$ and $v>1$, correspond to the Pöschl-Teller potential

$$
V_{P T}\left(x, 2,\left\{u+\frac{1}{2}, v+\frac{1}{2}\right\}\right)=\frac{1}{4}\left[\frac{u(u-1)}{\sin ^{2} \frac{x}{2}}+\frac{v(v-1)}{\cos ^{2} \frac{x}{2}}\right]-\frac{1}{4}(u+v)^{2},
$$

with an infinite non-degenerate spectrum $(n \in \mathbf{N})$.

(iii) $a=-2$ and $b=2 l+1$, with $l \in \mathbf{N}$, correspond to the Morse potential

$$
V_{M}(x,-2,2 l+1)=\mathrm{e}^{-2 x}-(2 l+3) \mathrm{e}^{-x}+(l+1)^{2},
$$

with an finite non-degenerate spectrum $(n=0,1, \cdots, l)$.

\subsection{Isospectral Hamiltonians}

The various isospectral Hamiltonians occuring in (5) are easily deduced from Eq. (6). This gives

$$
\begin{gathered}
H_{k-s} \equiv H_{k-s}(N, a, b)=(k-1) \times \\
\times\left[F\left(N, a, b-\frac{1}{2} k a+a+s a\right)+\frac{1}{6}(k-2)(k a-3 b)+\frac{1}{2} s(s-k+1) a+s b\right], \quad s \in S_{0} .
\end{gathered}
$$


Thus, the isospectral Hamiltonians are linked by

$$
H_{k-s}(N, a, b)=H_{0}(N, a, b+s a)+\frac{1}{2}(k-1) s(s a-a+2 b), \quad s \in S_{0},
$$

a relation of central importance, in the $k$-SUSYQM context, for the derivation of the translational shape invariance condition.

Let us denote by $V_{k} \equiv V_{0}, V_{k-1}, \cdots, V_{1}$ the potentials (in $x$-representation) associated with the isopectral Hamiltonians $H_{0}, H_{k-1}, \cdots, H_{1}$, respectively. In other words, we set

$$
H_{k-s}(N, a, b) \equiv-\frac{d^{2}}{d x^{2}}+V_{k-s}(x, a, b), \quad s \in S_{0}
$$

By using Eq. (12), we immediately get the recurrence relation

$$
V_{k-s}(x, a, b)=V_{0}(x, a, b+s a)+\frac{1}{2}(k-1) s(s a-a+2 b), \quad s \in S_{0},
$$

which may be considered as the $k$-SUSYQM version of the translational shape invariance condition for ordinary SUSY (see Eq. (1)).

By way of illustration, Eq. (13) yields the following results.

(i) For the harmonic oscillator system:

$$
V_{k-s}(x, 0,1)=x^{2}+\frac{1}{2}(k-1)(2 s-k+2) .
$$

(ii) For the Pöschl-Teller system:

$$
\begin{gathered}
V_{k-s}\left(x, 2,\left\{u+\frac{1}{2}, v+\frac{1}{2}\right\}\right)=\frac{1}{4}\left[\frac{\left(u+s+1-\frac{k}{2}\right)\left(u+s-\frac{k}{2}\right)}{\sin ^{2} \frac{x}{2}}+\frac{\left(v+s+1-\frac{k}{2}\right)\left(v+s-\frac{k}{2}\right)}{\cos ^{2} \frac{x}{2}}\right] \\
-\frac{1}{4}(u+v+2 s+2-k)^{2}+\frac{1}{6}(k-1)(k-2)(2 k-3 u-3 v-3)+(k-1) s(s-k+u+v+2) .
\end{gathered}
$$

(iii) For the Morse system:

$$
\begin{gathered}
V_{k-s}(x,-2,2 l+1)=\mathrm{e}^{-2 x}-(2 l+k+1-2 s) \mathrm{e}^{-x}+\frac{1}{4}(2 l+k-2 s)^{2} \\
-\frac{1}{6}(k-1)(k-2)(2 k+6 l+3)+(k-1) s(k-s+2 l)
\end{gathered}
$$

In the case $k=2$ and $s=0$, Eqs. (14), (15) and (16) reduce to Eqs. (9), (10) and (11), respectively.

\section{CYCLIC SHAPE INVARIANT POTENTIALS}

\subsection{Structure function}

In this section, we assume that $f_{s}(N)$ is independant of $N$, i.e.,

$$
f_{s}(N)=f_{s} \Rightarrow\left[X_{-}, X_{+}\right]=\sum_{s=0}^{k-1} f_{s} \Pi_{s}
$$


(The paradigmatic case of the harmonic oscillator corresponds to $f_{s}=$ constant for any $s$ in $S_{0}$.)

It is convenient to write the integer $n$ occurring in $|n, s\rangle$ as $n=k p+t$ with $p \in \mathbf{N}$ and $t \in S_{0}$. Here, to adapt our construction to one-dimensional periodic potentials, we restrict the Hilbert-Fock space $\mathcal{H}$ to its subspace $\mathcal{G}=\left\{|k p+s, s\rangle: p\right.$ ranging; $\left.s \in S_{0}\right\}$. In addition, it is appropriate to denote the state $|k p+s, s\rangle$ as $\mid k p+s)$. Hence, the action of the number operator $N$ on the states $\mid k n+s)$ is given by

$$
N \mid k n+s)=(k n+s) \mid k n+s)
$$

and the grading operator $K$ can be identified, on the subspace $\mathcal{G}$, with the operator $q^{N}$ since

$$
\left.\left.\left.K \mid k n+s)=q^{s} \mid k n+s\right)=q^{k n+s} \mid k n+s\right)=q^{N} \mid k n+s\right) .
$$

From Eq. (7), it can be shown

$$
F_{s}(N)=g_{0} N+\sum_{t=1}^{k-1} g_{t} \frac{1-q^{s t}}{1-q^{t}}
$$

where

$$
g_{t}=\frac{1}{k} \sum_{s=0}^{k-1} p^{s t} f_{s}, \quad t \in S_{0}
$$

Thus, the action of

$$
X_{+} X_{-}=\sum_{t=0}^{k-1} g_{t} \frac{1-q^{N t}}{1-q^{t}}
$$

on the space $\mathcal{G}$ reads

$$
\left.\left.X_{+} X_{-} \mid k n+s\right)=\left(n \sum_{i=0}^{k-1} f_{i}+\sum_{i=0}^{s-1} f_{i}\right) \mid k n+s\right) .
$$

The spectrum of $X_{+} X_{-}$is periodic and can be seen as a superposition of identical blocks. For a given block, the various gaps between the consecutive eigenvalues are

$$
f_{0}, f_{1}, \cdots, f_{k-1} .
$$

The first block (corresponding to $n=0$ ) has the following nonzero eigenvalues

$$
E_{1}=f_{0}, E_{2}=f_{0}+f_{1}, \cdots, E_{k}=f_{0}+f_{1}+\cdots+f_{k-1},
$$

while the second block (corresponding to $n=1$ ) has the eigenvalues

$$
E_{k+1}=E_{k}+f_{0}, E_{k+2}=E_{k}+f_{0}+f_{1}, \cdots, E_{2 k}=E_{k}+f_{0}+f_{1}+\cdots+f_{k-1},
$$

and so on for the subsequent blocks corresponding to $n=2,3, \cdots$ (the eigenvalue for the ground state is $\left.E_{0}=0\right)$. In other words, in the $(n+1)^{\text {th }}$ block the parameter $f_{s}$ is the difference between the eigenvalues for $\mid k n+s+1)$ and $\mid k n+s)$. According to Eq. (17), the various eigenvalues are given by

$$
E_{k n+s}=n k g_{0}+\sum_{i=0}^{s-1} f_{i}, \quad n \in \mathbf{N} ; s \in S_{0} .
$$


Thus, each block has the length $k g_{0}$ which can be considered as the period of the cyclic spectrum.

At this level, it should be emphasized that our approach covers the one of Ref. 11 concerning the two-body Calogero-Sutherland model. The latter model corresponds to $k=2$. Consequently, the relevant Hilbert-Fock space is

$$
\mathcal{G}=\{\mid 2 n+s): n \in \mathbf{N} ; s=0,1\}
$$

and $X_{+} X_{-}$reads

$$
X_{+} X_{-}=\frac{1}{2}\left(f_{0}+f_{1}\right) N+\frac{1}{2}\left(f_{0}-f_{1}\right) \Pi_{1} .
$$

Equation (17) can then be particularized as

$$
\begin{gathered}
\left.\left.X_{+} X_{-} \mid 2 n\right)=n\left(f_{0}+f_{1}\right) \mid 2 n\right), \\
\left.\left.X_{+} X_{-} \mid 2 n+1\right)=\left[n\left(f_{0}+f_{1}\right)+f_{0}\right] \mid 2 n+1\right),
\end{gathered}
$$

in accordance with the results of Ref. 11. (Our parameters $f_{0}$ and $f_{1}$ read $f_{0}=\omega_{0}$ and $f_{1}=\omega_{1}$ in the notations of Ref. 11.)

It is interesting to note that that the spectrum of $X_{+} X_{-}$coincides with one of the Hamiltonian corresponding to the potential (in $x$-representation)

$$
V_{0}\left(x, f_{0}, f_{1}\right)=\frac{1}{16}\left(f_{0}+f_{1}\right)^{2} x^{2}+\frac{1}{4} \frac{\left(f_{0}-f_{1}\right)\left(3 f_{0}+f_{1}\right)}{\left(f_{0}+f_{1}\right)^{2}} \frac{1}{x^{2}}-\frac{1}{2} f_{1} .
$$

Furthermore, using the standard tools of ordinary SUSYQM, we get

$$
V_{1}\left(x, f_{0}, f_{1}\right)=\frac{1}{16}\left(f_{0}+f_{1}\right)^{2} x^{2}+\frac{1}{4} \frac{\left(f_{1}-f_{0}\right)\left(3 f_{1}+f_{0}\right)}{\left(f_{0}+f_{1}\right)^{2}} \frac{1}{x^{2}}+\frac{1}{2} f_{0},
$$

which corresponds to the operator $X_{-} X_{+}$.

For $k>2$, the derivation of analytical forms of the potentials exhibiting a cyclic spectrum was discussed in Ref. 12.

\subsection{Isospectral Hamiltonians}

Going back to the general case, the expressions for the isospectral Hamiltonians in (6) can be obtained from (5). This yields the relations

$$
H_{s} \equiv H_{s}\left(N,\left\{f_{s}\right\}\right)=(k-1) X_{+} X_{-}+\sum_{t=2}^{k-1}(1-t) f_{t}+(k-1) \sum_{t=s}^{k-1} f_{t}, \quad s \in S_{1} .
$$

These relations show that the spectra of the supersymmetric partner Hamiltonians $H_{0}$, $H_{1}, \cdots, H_{k-1}$ can be deduced from the one of $X_{+} X_{-}$given by Eq. (17). By combining the latter two relations, we obtain

$$
H_{k-s}\left(N,\left\{f_{s}\right\}\right)=H_{0}\left(N+s,\left\{f_{s}\right\}\right), \quad s \in S_{0},
$$

an important relation for the derivation of the cyclic shape invariance condition. 
From (20), we can prove that

$$
H_{k-s}\left(N,\left\{f_{s}\right\}\right)=H_{0}\left(N, h^{(s)}\left\{f_{s}\right\}\right)+\sum_{i=0}^{s-1} f_{i}, \quad s \in S_{0}
$$

with

$$
h^{(s)}=h \circ h \circ \cdots \circ h, \quad s \text { times, }
$$

where $h$ is the circular permutation

$$
h\left\{f_{s}\right\}=h\left\{f_{0}, f_{1}, \cdots, f_{k-2}, f_{k-1}\right\}=\left\{f_{1}, f_{2}, \cdots, f_{k-1}, f_{0}\right\}
$$

such that $h^{(k)}$ is the identity.

We continue with dynamical systems in one-dimensional space (coordinate $x$ ). Let us note $V_{0}\left(x,\left\{f_{s}\right\}\right)$ the potential associated with $H_{0}\left(N,\left\{f_{s}\right\}\right)$ :

$$
H_{0}\left(N,\left\{f_{s}\right\}\right) \equiv-\frac{d^{2}}{d x^{2}}+V_{0}\left(x,\left\{f_{s}\right\}\right)
$$

From Eq. (21), it is easy to check that the potential $V_{k-s}\left(x,\left\{f_{s}\right\}\right)$ associated with the Hamiltonian $H_{k-s}\left(N,\left\{f_{s}\right\}\right)$ can be obtained via

$$
V_{k-s}\left(x,\left\{f_{s}\right\}\right)=V_{0}\left(x, h^{(s)}\left\{f_{s}\right\}\right)+\sum_{i=0}^{s-1} f_{i}, \quad s \in S_{0},
$$

to be compared with the cyclic shape invariance condition for ordinary SUSY (see Eq. (1) and Refs. 11 and 12).

As an example, for $k=2$, Eq. (22) leads to

$$
V_{1}\left(x,\left\{f_{0}, f_{1}\right\}\right)=V_{0}\left(x,\left\{f_{1}, f_{0}\right\}\right)+f_{0},
$$

a relation satisfied by Eqs. (18) and (19) for the Calogero-Sutherland potential.

\section{CONCLUDING REMARKS}

It was shown in the present paper how to tackle $k$-fractional SUSYQM through a $Z_{k}$-graded Weyl-Heisenberg algebra, noted $W_{k}$ with $k=3,4, \cdots$ (the case $k=2$ corresponding to ordinary SUSYQM). From the generators of this algebra, it was possible to find several realizations of $k$-fractional supersymmetric dynamical systems. Each system was characterized by a $k$-fractional supersymmetric Hamiltonian which gave rise to a hierarchy of $k$ isospectral Hamiltonians $H_{k-s}$ with $s \in S_{0}$. Two special cases of algebra $W_{k}$ were examined. They both led to $k$-fractional isospectral Hamiltonians, the potentials of which are connected by a recurrence relation that reflects a (translational or cyclic) shape invariance condition.

As a conclusion, $k$-fractional SUSYQM developed in the framework of algebra $W_{k}$ turns out to be a useful tool to generate a hierarchy of $k$ isospectral Hamiltonians linked by a translational or cyclic invariance condition.

A brief comparison with the results given by ordinary SUSYQM is in order. For $k=2$, the hierarchy of Hamiltonians reduces to a pair of isospectal Hamiltonians. Therefore, 
in order to generate a hierarchy of $k$ isospectal Hamiltonians, it is necessary to apply ordinary SUSYQM repeatedly. This is no longer the case for $k$-fractional SUSYQM since the hierarchy of $k$ isospectral Hamiltonians is generated at once. The equivalence between the approaches via ordinary SUSYQM applied repeatedly and $k$-fractional SUSYQM is ensured by the fact that $k$-SUSYQM can be seen as a superpostion of $k-1$ copies of ordinary SUSYQM. ${ }^{22}$

To close this paper, let us offer two remarks. First, it is worthwhile to mention that our approach to $k$-SUSYQM by means of algebra $W_{k}$ can be applied to other potentials. For instance, by taking $X_{+} X_{-} \equiv F(N, a, b, c)$, where the structure function $F$ is given by

$$
F(N, a, b, c)=\frac{1}{2} a N(N-1)+b N+c \frac{1}{(N+1)^{2}},
$$

it might be possible to describe potentials involving a Coulombic part. Along this vein, a $k$-SUSYQM study to the effective screened potential, ${ }^{40}$ singular inverse-power potentials, ${ }^{41}$ and non-central potentials ${ }^{42}$ could be fruitful. Second, it would interesting to examine the hidden supersymmetries exhibited by the Aharanov-Bohm, Dirac delta, and Pöschl-Teller potentials $^{34-36}$ in the light of our approach to $k$-fractional SUSYQM. For this purpose, the connection between ordinary SUSYQM, possibly in a $q$-deformed approach, ${ }^{43}$ and $k$-SUSYQM ${ }^{22}$ should play a central role.

\section{Acknowledgment}

One of the authors (M.D.) would like to thank the Groupe de Physique Théorique of the Institut de Physique Nucléaire de Lyon for the kind hospitality extended to him during July 2006. 


\section{References}

[1] J. Wess and B. Zumino, Nucl. Phys. B 70, 39 (1974).

[2] E. Witten, Nucl. Phys. B 188, 513 (1981).

[3] G. Junker, Supersymmetric Methods in Quantum and Statistical Physics (Springer, Berlin, 1996).

[4] B. K. Bagchi, Supersymmetry in Quantum and Classical Mechanics (Chapman and Hall, London, 2000).

[5] F. Cooper, A. Khare and U. Sukhatme, Supersymmetry in Quantum Mechanics (World Scientific, Singapore, 2001); Phys. Rep. 251, 268 (1995).

[6] L. Gendenshtein, JETP Lett. 38, 356 (1983).

[7] F. Cooper, J. N. Ginocchio and A. Khare, Phys. Rev. D 36, 2458 (1987).

[8] C. Chuan, J. Phys. A 24, L1165 (1991).

[9] A. Khare and U. Sukhatme, J. Phys. A 26, L901 (1993).

[10] D. T. Barclay, R. Dutt, A. Gangopadhyaya, A. Khare, A. Pagnamenta and U. Sukhatme, Phys. Rev. A 48, 2786 (1993).

[11] A. Gangopadhyaya and U. P. Sukhatme, Phys. Lett. A 224, 5 (1996).

[12] U. P. Sukhatme, C. Rasinariu and A. Khare, Phys. Lett. A 234, 401 (1997).

[13] V. A. Rubakov and V. P. Spiridonov, Mod. Phys. Lett. A 3, 1337 (1988).

[14] J. Beckers and N. Debergh, Mod. Phys. Lett. A 4, 1209 (1989); Nucl. Phys. B 340, 767 (1990); N. Debergh, J. Math. Phys 34, 1270 (1993); J. Phys. A. 26, 7219 (1993); 27, L213 (1994).

[15] A. Khare, J. Phys. A 25, L749 (1992); J. Math. Phys. 34, 1277 (1993).

[16] A. T. Filippov, A. P. Isaev and A. B. Kurdikov, Mod. Phys. Lett. A 7, 2129 (1992); Int. J. Mod. Phys. A 8, 4973 (1993).

[17] S. Durand, Mod. Phys. Lett. A 7, 2905 (1992); Phys. Lett. B 312, 115 (1993); Mod. Phys. Lett. A 8, 2323 (1993).

[18] S. Klishevich and M. S. Plyushchay, Mod. Phys. Lett. A 14, 2379 (1999).

[19] M. Daoud, Y. Hassouni and M. Kibler, in Symmetries in Science X, edited by B. Gruber and M. Ramek (Plenum, New York, 1998); Yad. Fiz. 61, 1935 (1998).

[20] M. Daoud and M. Kibler, in Symmetry and Structural Properties of Condensed Matter, edited by T. Lulek, B. Lulek and A. Wal (World Scientific, Singapore, 2001); in Proceedings of the Sixth International Wigner Symposium (Bogazici Univ. Press, Istanbul, Turkey, 2002). 
[21] H.-Y. Pan and Z. S. Zhao, Phys. Lett. A 312, 1 (2003).

[22] M. Daoud and M. Kibler, Phys. Lett. A 321, 147 (2004).

[23] C. Ahn, D. Bernard and A. LeClair, Nucl. Phys. B 346, 409 (1990); A. LeClair and C. Vafa, Nucl. Phys. B 401, 413 (1993).

[24] R. Kerner, J. Math. Phys. 33, 403 (1992).

[25] J. L. Matheus-Valle and M. A. R.-Monteiro, Mod. Phys. Lett. A 7, 3023 (1992); Phys. Lett. B 300, 66 (1993); L. P. Collato and J. L. Matheus-Valle, J. Math. Phys. 37, 6121 (1996).

[26] E. H. Saidi, M. B. Sedra and J. Zerouaoui, Class. and Quant. Gravity 7, 1567 (1995).

[27] N. Mohammedi, Mod. Phys. Lett. A 10, 1287 (1995).

[28] J. A. de Azcárraga and A. J. Macfarlane, J. Math. Phys. 37, 1115 (1996).

[29] A. Perez, M. Rausch de Traubenberg and P. Simon, Nucl. Phys. B 482, 325 (1996); N. Fleury and M. Rausch de Traubenberg, Mod. Phys. Lett. A 11, 899 (1996); M. Rausch de Traubenberg and M. J. Slupinski, Mod. Phys. Lett. A 12, 3051 (1997); M. Rausch de Traubenberg and P. Simon, Nucl. Phys. B 517, 485 (1998); M. Rausch de Traubenberg and M. J. Slupinski, J. Math. Phys. 41, 4556 (2000).

[30] A. Mostafazadeh, Int. J. Mod. Phys. A 11, 1057, 2941, 2957 (1996); K. Aghababaei Samani and A. Mostafazadeh, Nucl. Phys. B 595, 467 (2001).

[31] H. Ahmedov and Ö. F. Dayi, J. Phys. A 32, 6247 (1999).

[32] M. S. Plyushchay, Ann. Phys. 245, 339 (1996); Mod. Phys. Lett. A 11, 2953 (1996); Nucl. Phys. B 491, 619 (1997); Mod. Phys. Lett. A 12, 1153 (1997).

[33] M. Plyushchay, Int. J. Mod. Phys. A 15, 3679 (2000).

[34] F. Correa, M. A. del Olmo and M. S. Plyushchay, Phys. Lett. B 628, 157 (2005).

[35] F. Correa and M. S. Plyushchay, hep-th/0605104.

[36] F. Correa, L.-M. Nieto and M. S. Plyushchay, hep-th/0608096.

[37] M. Daoud and M. Kibler, Phys. Part. Nuclei (Supp. 1) 33, S43 (2002); Int. J. Quant. Chem. 91, 551 (2003); M. R. Kibler and M. Daoud, in Fundamental World of Quantum Chemistry, edited by E. J. Brändas and E. S. Kryachko (Kluwer, Dordrecht, 2004).

[38] A. Chenaghlou and H. Fakhri, Int. J. Mod. Phys. A 18, 939 (2003).

[39] M. Stos̃ić and R. Picken, math-phys/0407019.

[40] Shang-Wu Qian, Bo-Wen Huang and Zhi-Yu Gu, New J. Phys. 4, 13.1 (2002).

[41] B. Gönül, O. Özer, M. Koçak, D. Tutcu and Y. Cançelik, J. Phys. A 34, 8271 (2001). 
[42] M. Koçak, I. Zorba and B. Gönül, Mod. Phys. Lett. A 17, 2127 (2002).

[43] A. N. F. Aleixo, A. B. Balantekin and M. A. Cândido Ribeiro, J. Phys. A 36, 11631 (2003). 